\title{
Structure-Related Effects of Pyrethroid Insecticides on the Lateral-Line Sense Organ and on Peripheral Nerves of the Clawed Frog, Xenopus laevis
}

\author{
Henk P. M. Vijverberg, Ge S. F. Ruigt, and Joep van den Bercken ${ }^{1}$ \\ Department of Veterinary Pharmacology, Pharmacy and Toxicology, University of Utrecht, \\ Biltstraat 172, 3572 BP Utrecht. The Netherlands
}

Received March 22, 1982; accepted July 16, 1982

\begin{abstract}
The effects of seven different pyrethroid insecticides on the lateral-line sense organ and on peripheral nerves of the clawed frog, Xenopus laevis, were investigated by means of electrophysiological methods. The results show that two classes of pyrethroid can be clearly distinguished. (i) Pyrethroids without an $\alpha$-cyano group (permethrin, cismethrin, and bioresmethrin). These noncyano pyrethroids induce short trains of nerve impulses in the lateral-line sense organ. In peripheral nerve branches they induce a depolarizing afterpotential and repetitive firing. These effects are very similar to those previously reported for allethrin. (ii) Pyrethroids with an $\alpha$-cyano3-phenoxybenzyl alcohol (cypermethrin, fenpropathrin, deltamethrin, and fenvalerate). In the lateral-line sense organ these $\alpha$-cyano pyrethroids induce very long trains of nerve impulses which may last for seconds and may contain hundreds or even thousands of impulses. The $\alpha$-cyano compounds do not cause repetitive activity in peripheral nerves. Instead they induce a quickly reversible, stimulus frequency-dependent suppression of the action potential. Since the chemical structure of cypermethrin differs from that of permethrin only in the $\alpha$-cyano group and because all four $\alpha$-cyano compounds act in a very similar way, it is concluded that the $\alpha$-cyano substituent is responsible for the large differences in neurotoxic effects. In the lateral-line sense organ the duration of nerve impulse trains induced by the noncyano as well as the $\alpha$-cyano pyrethroids increases dramatically when the temperature is lowered. Further, in sensory fibers the effects of both classes of pyrethroid on the nerve action potential are more pronounced compared to their effects on motor fibers. It is argued that the different neurotoxic effects reported here originate from a common mechanism of action of pyrethroids, which is a prolongation of the transient increase of sodium permeability of the nerve membrane associated with excitation.

It is concluded that the sodium channel in the nerve membrane is the major target site of noncyano and $\alpha$-cyano pyrethroids.
\end{abstract}

\section{INTRODUCTION}

Pyrethroid insecticides are generally recognized as typical neurotoxicants that act directly on excitable tissues $(1-3)$. Farly electrophysiological investigations into the neurotoxic action of allethrin have shown that this compound induces a depolarizing afterpotential following the action potential together with repetitive activity in nerve fibers. A brief electrical stimulus applied to the poisoned nerve produces a train of impulses instead of a single nerve impulse (4, 5). A large number of pyrethroids have been reported to induce repetitive activity

To whom all correspondence should be sent. in isolated nerve preparations of invertebrates and vertebrates, including mammals $(5-9)$. This effect has been observed in the peripheral as well as in the central nervous system $(9-12)$. In particular, sense organs $(13,14)$ and presynaptic nerve endings $(12$, $15,16)$ show repetitive activity after treatment with pyrethroids. A genuine effect of pyrethroids on chemical neurotransmission has not been reported so far.

Voltage clamp experiments have revealed that allethrin and some other pyrethroids cause a prolongation of the transient increase in sodium permeability associated with depolarization of the nerve membrane $(17-20)$. The prolonged sodium current is 
thought to be directly responsible for the repetitive activity induced by pyrethroids $(2,13)$. It has also been reported that pyrethroids, especially at high concentrations, may block nervous activity by suppressing the sodium and potassium permeability of the nerve membrane $(21,22)$.

The intensity of repetitive activity induced by different pyrethroids varies considerably, but a clear correlation between structure and activity has not yet been established $(6,7,23,24)$. Recently we have found that deltamethrin, an $\alpha$-cyano-3phenoxybenzyl ester, causes intense repetitive activity in the lateral-line sense organ of Xenopus laevis but fails to induce repetitive firing in myelinated nerve fibers (25). According to the symptoms of poisoning in the rat two classes of pyrethroid can be distinguished which to a large extent coincide with the presence of an $\alpha$-cyano group (23). Recently a similar division of pyrethroids into two classes was made based on the ability to induce repetitive nerve activity $(24,26)$. Because of the remarkable difference in poisoning symptoms and the apparent failure of $\alpha$-cyano pyrethroids to induce repetitive activity in cockroach sensory nerves it was suggested that these two classes of pyrethroid have a different primary mechanism of action (24).

In the present study on the peripheral nervous system of Xenopus laevis large differences in neurotoxic effects between $\alpha$-cyano and noncyano pyrethroids are observed, which are due to the presence of the $\alpha$-cyano group. The results, however, are in keeping with the notion that both classes of pyrethroid primarily affect nerve membrane sodium channels in essentially the same way.

\section{MATERIALS AND METHODS}

The experiments were performed on the lateral-line sense organ and on peripheral nerves of small specimens $(6-9 \mathrm{~cm})$ of the clawed frog, Xenopus laevis. Standard electrophysiological methods were used to study the effects of pyrethroids on spon- taneous afferent activity of the lateral-line organ and on electrically evoked compound action potentials of peripheral nerve branches.

Lateral-line sense organ. A piece of skin containing several lateral-line organs was removed from the dorsolateral side of the frog and was fixed between two Perspex rings. The skin was placed with the outer surface downward in a petri dish filled with tap water. The petri dish was mounted on a Peltier element for temperature control and the temperature of the tap water was kept constant $\left( \pm 0.5^{\circ} \mathrm{C}\right)$ in a range of $8-22^{\circ} \mathrm{C}$. The imner side of the skin was covered with Ringer's solution. The nerve innervating the lateral line was dissected free from the skin in such a way that one lateral-line organ was left connected, which is innervated by only two afferent nerve fibers. Spontaneous afferent nerve activity was recorded by means of a silver wire electrode. Further details on preparation and recording have been described previously (27). The two types of nerve impulses, each originating from one of the afferent nerve fibers, were separated by amplitude discrimination and nerve impulse intervals from one fiber were measured with a timer counter that was interfaced to a minicomputer system. So all data reported here refer to single-fiber activity. Data analysis was performed on line, while a parallel recording of nerve activity was made on magnetic tape for later reviewing.

Peripheral nerves. Excised sciatic nerves were mounted in a moist chamber across silver wire electrodes. The nerve was stimulated by brief square pulses applied through an optical isolation unit and monophasic compound action potentials were recorded. The signals were amplified, displayed on an oscilloscope, and photographed. Between measurements the nerves were immersed in Ringer's solution. For experiments with motor and sensory nerve fibers the tibial nerve was dissected together with the sensory branch that innervates the skin (ramus cutancous cruris 
posterior) and with the motor branch that innervates the head of the gastrocnemic muscle. Activity from these nerve branches was recorded using a suction pipet electrode. Nerve experiments were performed at a room temperature of $19-23^{\circ} \mathrm{C}$.

Treatment with pyrethroids. Pyrethroids were dissolved in ethanol, except for deltamethrin that was dissolved in acetone. A small amount of stock solution was squirted through a hypodermic needle in water or in Ringer's solution to produce a fine suspension. In the experiments with the lateralline sense organ the pyrethroids were either added to the water in which the animal was kept (in vivo exposure), or to the water at the outer surface of the isolated skin preparation (in vitro exposure). In the in vivo experiments the animal was killed before serious symptoms of poisoning developed and a lateral-line preparation was set up. Excised nerves were immersed in Ringer's solution containing the desired concentration of pyrethroid. The final amount of solvent was always less than $0.5 \%(\mathrm{v} / \mathrm{v})$ which was without effect in control experiments.

Chemicals. The Ringer's solution contained (in $\mathrm{m} M$ ): $\mathrm{NaCl}, 116 ; \mathrm{KCl}, 2.4 ; \mathrm{CaCl}_{2}$, 2.0 , and Hepes buffer, 3 ; pH was adjusted to 7.3 .

The following pyrethroids were used:

permethrin [3-phenoxybenzyl-(1RS,3RS)-3-

(2,2-dichlorovinyl)-2,2-dimethylcyclopropanecarboxylate (WL 43479)]

cismethrin [5-benzyl-3-furylmethyl-(1R,3S)chrysanthemate (NRDC 119)]

bioresmethrin [5-benzyl-3-furylmethyl-( $1 R$,

$3 R$ )-chrysanthemate (NRDC 107)]

cypermethrin $[(R S)-\alpha$-cyano-3-phenoxybenzyl-(1R, 3RS)-3-(2,2-dichlorovinyl)-2,2dimethylcyclopropanecarboxylate (WL 43476)]

fenpropathrin [(RS)- $\alpha$-cyano-3-phenoxyben-

zyl-2,2,3,3-tetramethylcyclopropanecarboxylate (S 3206)]

fenvalerate $[(R S)$ - $\alpha$-cyano-3-phenoxybenzyl-(RS)-2-(4-chlorophenyl)-3-methylbutyrate (WL 43775)] deltamethrin $[(S)$ - $\alpha$-cyano-3-phenoxybenzyl(1R,3S)-3-(2,2-dibromovinyl)-2,2-dimethylcyclopropanecarboxylate (NRDC 161)]

\section{RESULTS}

\section{Lateral-Line Sense Organ}

A typical characteristic of the lateral-line sense organ is its spontaneous afferent activity which occurs in the absence of any mechanical stimulation (28). In control experiments single afferent nerve impulses occurred at irregular intervals with a mean rate of $10-25 \mathrm{~Hz}$ at room temperature, as illustrated in Fig. 1A. The pyrethroids caused pronounced repetitive activity in the lateral-line sense organ and after treatment with the insecticides spontaneous trains of nerve impulses were observed alternated by silent intervals with occasionally a few single-nerve impulses. The number of nerve impulses per train markedly varied for the different compounds and also depended strongly on temperature as described below. The intervals between the trains increased with increasing train duration. The frequency of the nerve impulses during the trains was between 100 and $200 \mathrm{~Hz}$ at room temperature. The small differences in nerve impulse frequency during the trains for the various pyrethroids were not investigated further. The delay between exposure to pyrethroids and the onset of repetitive activity varied considerably for the different compounds and ranged from 1 to $24 \mathrm{hr}$. Cypermethrin and fenvalerate even failed to induce repetitive activity in the time span of the in vitro experiments $(4-5 \mathrm{hr})$. However, significant differences in repetitive activity between preparations that were treated in vivo or in vitro with permethrin or cismethrin were not observed. Therefore, the effects of the pyrethroids on the lateral-line organ are compared here independent of the way of application.

Comparison of average numbers of impulses per train at $10^{\circ} \mathrm{C}$ revealed large differences between the various pyrethroids. Lateral-line preparations exposed to $5 \times$ 
A

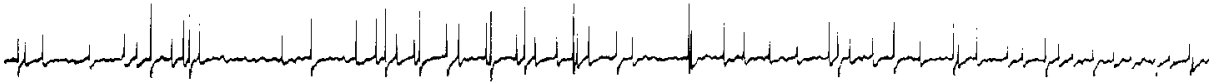

B

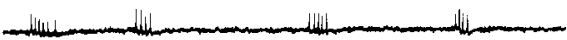

D

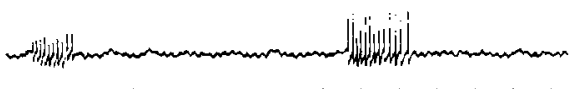

C

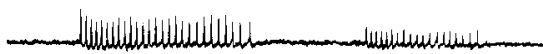

E

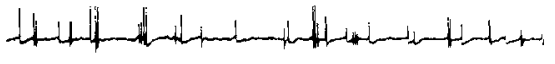

$\mathbf{F}$

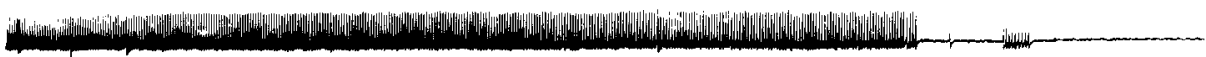

G



FIG. 1. Effects of various pyrethroids on the spontaneous afferent activity of the lateral-line sense organ. Spontaneous activity in a control preparation at $19^{\circ} \mathrm{C}(A)$, repetitive nerve impulse trains induced by permethrin at $15(B)$ and $8^{\circ} \mathrm{C}(\mathrm{C})$, cismethrin at $17^{\circ} \mathrm{C}(\mathrm{D})$, bioresmethrin at $10^{\circ} \mathrm{C}(\mathrm{E})$, cypermethrin at $14^{\circ} \mathrm{C}(\mathrm{F})$, and fenpropathrin at $13^{\circ} \mathrm{C}(\mathrm{G})$ all at a concentration of $5 \times 10^{-6} \mathrm{M}$. Time calibration, 100 msecidivision; upper applies to $A-E$, lower to $F$ and $G$. In $G, 8.3$ sec of the nerve impulse train have been omitted.

$10^{-6} \mathrm{M}$ permethrin in vitro for $3 \mathrm{hr}$ showed short trains of nerve impulses (Fig. 1B). The average number of impulses per train ranged from 6 to 11 with a mean of 7 in three experiments. After in vivo treatment with $10^{-5} M$ permethrin for $5 \mathrm{hr}$ very similar repetitive activity in the lateral-line sense organ was observed.

Cismethrin at a concentration of $1-5 \times$ $10^{-6} M$ for $1-3 \mathrm{hr}$ caused repetitive activity that was somewhat more pronounced than after treatment with permethrin (Fig. 1D). In three in vivo and three in vitro experiments nerve impulse trains were observed which contained an average number of impulses per train ranging from 5 to 80 with a mean of 25 impulses per train. One animal that was exposed to $2 \times 10^{-6} M$ cismethrin for $220 \mathrm{~min}$ showed hyperexcitation and convulsions, but in the other in vivo experiments the animals were killed before gross behavioral symptoms became apparent.
Bioresmethrin at $5 \times 10^{-6} M$ for $2-3 \mathrm{hr}$ in vivo caused only weak repetitive activity in the lateral-line sense organ (Fig. 1E). The average number of impulses per train ranged from 2 to 6 with a mean of 3 in seven experiments. Thus bioresmethrin is much less efficacious in producing repetitive activity in the lateral-line organ than its diastereoisomer cismethrin.

Treatment with the $\alpha$-cyano-3-phenoxybenzyl pyrethroids also caused repetitive activity in the lateral-line sense organ, but the trains of impulses lasted much longer compared to the trains induced by the noncyano compounds and one train could last for seconds and could contain hundreds or even thousands of impulses.

Cypermethrin, the $\alpha$-cyano analog of permethrin, induced long-lasting trains of nerve impulses in lateral-line preparations from animals treated with $5 \times 10^{-6} M$ for a period of $4 \mathrm{hr}$ in vivo (Fig. 1F). The average 
number of impulses per train ranged from 130 to 790 with a mean of 340 in seven experiments.

Fenpropathrin acted more rapidly and after $1-1.5 \mathrm{hr}$ of in vitro exposure to $5 \times$ $10^{-6} M$ trains of impulses were recorded with an average number of impulses per train that ranged from 28 to 2450 with a mean of 530 in six experiments (Fig. 1G).

Deltamethrin produced similarly long trains of impulses in five preparations treated in vitro with $1-5 \times 10^{-6} M$ for $3 \mathrm{hr}$. In three experiments the average number of impulses per train at $13^{\circ} \mathrm{C}$ ranged from 370 to 890 with a mean of 528 .

Fenvalerate acted very slowly and animals had to be treated in vivo with $5 \times 10^{-6}$ $M$ for up to $23 \mathrm{hr}$ before the lateral-line organ showed repetitive activity. In two experiments the average number of impulses per train at $10^{\circ} \mathrm{C}$ ranged from 300 to 340 .

These results show that all four $\alpha$-cyano pyrethroids caused long-lasting trains of repetitive activity in the lateral-line sense organ with a mean number of impulses per train that is an order of magnitude higher than that after treatment with the noncyano compounds, including allethrin (5). At the concentrations used a suppressive effect of the pyrethroids on spontaneous activity was not detected.

\section{Effects of Temperature}

In control preparations the mean rate of spontaneous activity of the lateral-line organ gradually decreased when the temperature was lowered from 22 to $8^{\circ} \mathrm{C}$. In preparations which showed repetitive activity the number of impulses per train and train duration increased dramatically with cooling, whereas the frequency of impulses during the train and the frequency of the trains gradually decreased. This effect was readily reversed when the temperature was raised. Figures $1 B$ and $C$ show the effect of cooling in a lateral-line preparation treated with permethrin. The relation between temperature and the average number of impulses per train in three lateral-line preparations treated with the noncyano pyrethroids permethrin, cismethrin, and bioresmethrin is shown in Fig. 2A. At higher temperatures the number of impulses per train was reduced to a minimum of two. When the temperature was raised further the number of paired impulses gradually decreased and eventually the repetitive activity disappeared completely. A simi-
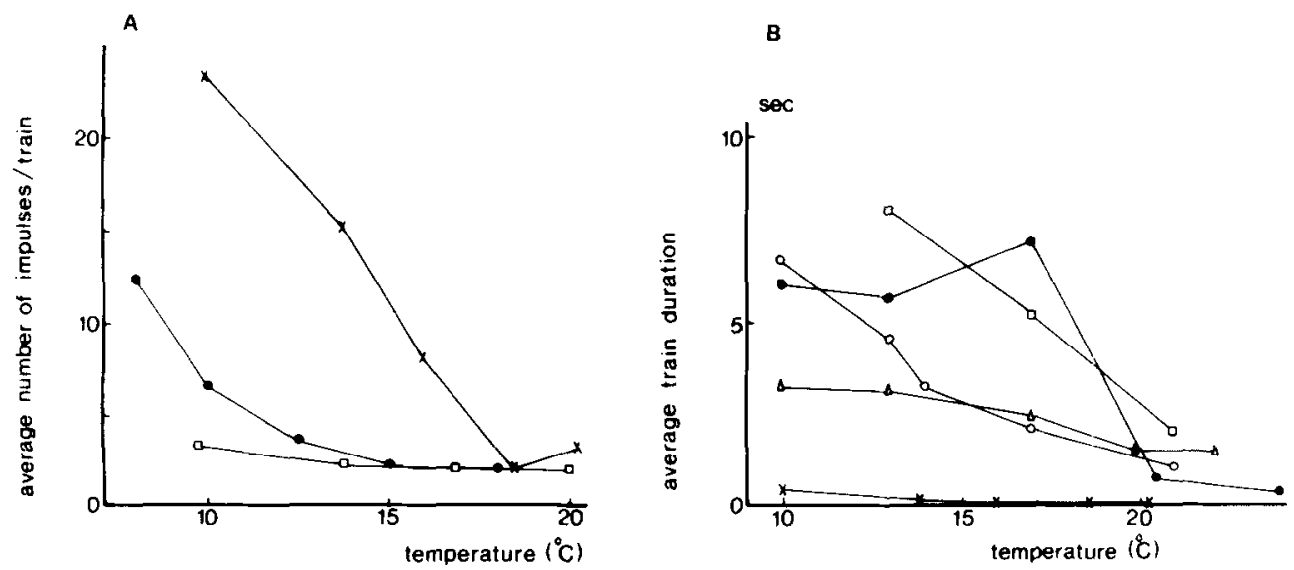

FIG. 2. Relation between temperature and average number of impulses per train for permethrin (๑), cismethrin $(X)$, and bioresmethrin $(\square)(A)$. Relation between temperature and average train duration for cypermethrin $(\triangle)$, fenpropathrin $(\bullet)$, deltamethrin $(\square)$ and fenvalerate $(O)(B)$. The average train duration after cismethrin $(X)$ from the same experiment as in $A$ is also depicted in $B$. All concentrations were $5 \times 10^{-6} \mathrm{M}$. 
lar negative correlation between temperature and repetitive activity in the lateralline sense organ has been reported before for allethrin (5).

For the $\alpha$-cyano pyrethroids the number of impulses per train and train duration also dramatically increased with cooling as described for the noncyano compounds. Figure $2 \mathrm{~B}$ shows the relation between temperature and the average train duration after treatment with cypermethrin, fenpropathrin, deltamethrin, and fenvalerate. For comparison, the average train duration after cismethrin from the same experiment as depicted in Fig. 2A is also presented. At temperatures below $10^{\circ} \mathrm{C}$ the duration of the nerve impulse trains further increased, but the occurrence of trains became very irregular and they were separated by very long silent periods. Nerve impulse trains that lasted for more than $30 \mathrm{sec}$ and which contained thousands of impulses were occasionally observed.

\section{Peripheral Nerves}

The effects of pyrethroids on the compound action potential of peripheral nerves differed for noncyano and $\alpha$-cyano compounds. Permethrin, cismethrin and bioresmethrin at a concentration of $5 \times 10^{-6}$ $M$ caused repetitive activity within the first $2 \mathrm{hr}$ of exposure. The nerve action potential was followed by a depolarizing afterpotential and repetitive afterdischarges were superimposed on this afterpotential. Dependent on the degree of synchronization of afterdischarges sometimes large potential oscillations following the action potential were observed. Figure $3 \mathrm{~A}$ shows a compound action potential of a sciatic nerve treated with cismethrin. The same concentration of bioresmethrin caused only small effects.

In the sensory branch of the tibial nerve pronounced repetitive activity was observed after treatment with noncyano pyrethroids, whereas in the motor branch hardly any repetitive activity was observed. This marked difference is shown for per- methrin in Figs. 3B and C. These effects closely resemble those previously reported for the noncyano pyrethroid allethrin (5).

Excised sciatic nerves exposed to cypermethrin, fenpropathrin, deltamethrin, or fenvalerate in concentrations up to $10^{-5}$ $M$ for more than $24 \mathrm{hr}$ did not show repetitive activity after a single stimulus and a depolarizing afterpotential was not detected. However, when a short train of stimuli was applied the amplitude of the compound action potential gradually declined as shown in Fig. 3D for fenpropathrin. This effect was neither observed in control nerves at the same stimulus frequency nor after the noncyano pyrethroids. The degree of suppression of the compound action potential increased with increasing stimulus frequency. The suppression was quickly reversed when the nerve was left unstimulated for a few seconds and the action potential fully regained its original amplitude. This stimulus-frequency-dependent suppression of the nerve action potential has been reported before for deltamethrin (25).

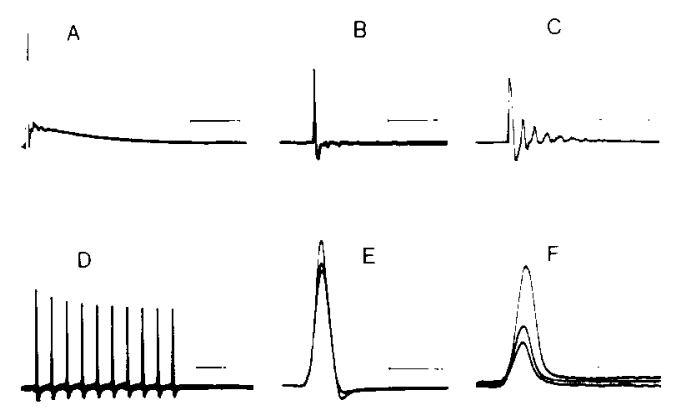

FIG. 3. Effects of various pyrethroids on the nerve action potential. Depolarizing afterpotential and repetitive activity in the excised sciatic nerve after $5 \times$ $10^{-6} \mathrm{M}$ cismethrin for $4 \mathrm{hr}(A)$. Repetitive activity after prolonged treatment with $I \times 10^{-5} \mathrm{M}$ permethrin $(22$ $h r)$ in the motor branch $(B)$ and in the sensory branch of the tibial nerve $(C)$. Stimulas-frequency-dependent suppression of the nerve action potential by $5 \times 10^{-6}$ $M$ fenpropathrin $(40$ min) in the sciatic nerve $(D)$ and after prolonged treatment with $1 \times 10^{-6} \mathrm{M}$ cypermethrin $(/ 9 \mathrm{hr})$ in the motor branch of the tibial nerve $(E)$ and in the sensory branch $(F)$. In $E$ and $F$, three stimuli were applied at a frequency of $5 \mathrm{~Hz}$. Time calibrations. $20 \mathrm{msec}(A-D)$ and $2 \mathrm{msec}(E, F)$. Temperature. $19-22^{\circ} \mathrm{C}$. 
At high stimulus frequencies the last action potential of a train was sometimes followed by a small depolarizing afterpotential and in some nerves treated with fenvalerate a few repetitive discharges of low amplitude occurred at the end of the train. A slowly progressing, irreversible suppression of the nerve action potential after treatment with the $\alpha$-cyano compounds was also observed, but this effect was quite distinct from the reversible, frequency-dependent suppression. In the sensory branch of the tibial nerve the frequency-dependent suppression of the action potential was more pronounced than in the motor branch as illustrated in Figs. $3 \mathrm{E}$ and $\mathrm{F}$.

\section{DISC USSION}

The present results demonstrate that, based on their effects on the peripheral nervous system of Xenopus laevis, the pyrethroids can be divided into noncyano and $\alpha$-cyano compounds. The afferent nerve impulse trains induced in the lateral-line sense organ by these two classes of pyrethroid differ strikingly in duration. Moreover, in peripheral nerves noncyano pyrethroids induce marked repetitive activity, whereas the $\alpha$-cyano compounds only cause a stimulus-frequency-dependent suppression of the nerve action potential. The difference in effect between permethrin and cypermethrin is of particular interest. These two pyrethroids have identical chemical structures, except that cypermethrin contains an $\alpha$-cyano group. This demonstrates that the large differences in effects between $\alpha$-cyano and noncyano pyrethroids are solely due to the presence of the $\alpha$-cyano group.

Because of the marked differences in neurotoxic effects it is not surprising that these two classes of pyrethroid produce quite different symptoms of poisoning in intact animals $(23,24)$. Verschoyle and Aldridge (23) have reported that the poisoning syndrome of the non-cyano pyrethroids in the rat is quite distinct from that of most $\alpha$-cyano pyrethroids, with the notable exception of fenpropathrin. This indicates that besides the effects on the peripheral nervous system described here, other factors may play an important role in the development of the poisoning syndrome of pyrethroids.

Recently Gammon et al. (24) have also distinguished between two classes of pyrethroid action in the cockroach, Periplaneta americana, based on symptomology and neurophysiological effects. Their type II pyrethroids which include cypermethrin, deltamethrin, and fenvalerate were characterized by the failure to produce repetitive firing in the cercal sensory nerve. Although this agrees with the absence of repetitive activity in frog nerves treated with $\alpha$-cyano pyrethroids, it contrasts sharply with the effects in the lateral-line sense organ. Prolonged trains of nerve impulses have also been reported in the tarsal nerve of Locusta migratoria after treatment with $\alpha$-cyano pyrethroids (28).

Voltage clamp experiments have revealed that pyrethroids primarily affect the sodium channels in the nerve membrane and cause a prolongation of the transient increase in sodium permeability associated with membrane depolarization (17-20). After treatment with pyrethroids the sodium current evoked by a step depolarization is followed by a prolonged sodium tail current after repolarization of the membrane. The prolonged sodium current is not only directly responsible for the depolarizing afterpotential following the nerve action potential, but also for the repetitive activity induced by pyrethroids $(2,13)$. Recently, we have investigated the effects of a number of $\alpha$-cyano and noncyano pyrethroids on sodium currents in Xenopus nodal membrane $(30,31)$. It was found that the $\alpha$-cyano pyrethroids induced a very long-lasting sodium tail current, whereas the tail current induced by the noncyano pyrethroids was at least one order of magnitude shorter. Moreover, lowering the temperature resulted in a considerable 
further prolongation of the sodium tail currents induced by both classes of pyrethroid. It, therefore, appears that the prolonged sodium tail currents induced by $\alpha$-cyano pyrethroids are associated with long-lasting trains of nerve impulses in the lateral-line sense organ, whereas noncyano compounds cause much shorter tail currents and short trains of nerve impulses. From the correlation between tail currents and nerve impulse trains it is concluded that the negative temperature coefficient of repetitive activity in the lateral-line organ is due to the further prolongation of the sodium tail current with cooling.

Experiments with single myelinated nerve fibers have shown that deltamethrin causes a small, but long-lasting, depolarizing afterpotential and that the frequency-dependent suppression of the action potential is brought about by a gradual depolarization of the nerve membrane due to summation of afterpotentials during train stimulation (25). Further, following a short depolarizing pulse deltamethrin caused a sodium tail current of low amplitude. The inability of $\alpha$-cyano pyrethroids to produce repetitive firing in peripheral nerves may, therefore, be explained by the fact that the amplitude of the sodium tail current induced by these compounds is too small to elicit a repetitive response in isolated nerve fibers. On the other hand, the more rapidly decaying sodium tail currents induced by noncyano compounds, which are of sufficient amplitude to elicit a repetitive response in sensory nerve fibers, cause a short afterpotential that does not lead to a sustained membrane depolarization during train stimulation. It has been suggested before that differences in kinetics of the sodium inactivation process between sensory and motor fibers are of critical importance for the generation of a repetitive response by pyrethroids $(13,16)$.

It seems unlikely that the prolonged trains of impulses in the lateral-line sense organ originate from an effect of the $\alpha$ cyano compounds on the synapse between the sensory hair cell and the afferent nerve ending, as suggested by Gammon et al. (24). Gaba (32) or glutamate (33) have been proposed as transmitter for this synapse. It has been shown that pyrethroids do neither affect glutamate potentials in locust muscle (6) nor the Gaba-mediated inhibitory transmission in rat spinal cord (34). In addition the present results show a gradual but no fundamental difference between nerve impulse trains induced by noncyano and $\alpha$-cyano compounds. It has also been demonstrated that pyrethroids cause repetitive activity in primary sense organs, e.g., the cutaneous touch receptor of the frog (35) and the crayfish stretch receptor (36); G. S. F. Ruigt, J. F. L. Klis, and J. van den Bercken (unpublished observations).

The intravenous toxicity of cismethrin in the rat is much higher than that of its trans-isomer bioresmethrin, although both compounds have about equal insecticidal activities (37). Gray et al. (37) have shown that this difference is most probably due to a difference in intrinsic neurotoxic activity. This finding is corroborated by the present observations in Xenopus. The effects of permethrin, cismethrin, and allethrin (5) do not grossly deviate from each other which indicates that the alcohol moiety of the pyrethroid molecule may vary considerably without large effects on neurotoxic activity. Also, the differences in the unsaturated side chain of the acid part of the pyrethroid molecule appear to be of minor influence as can be concluded from the similarity in action of cypermethrin, fenpropathrin, deltamethrin, and fenvalerate. However, the introduction of a cyano group at the $\alpha$ position of the 3-phenoxybenzyl alcohol greatly increases the degree of repetitive activity. This indicates that the central part of the pyrethroid molecule is intimately involved in the interaction with the nerve membrane sodium channel, although it remains an enigma why the cyano group has such a marked effect.

In summary, our results support the notion that the pyrethroids primarily interact 
with the sodium channels in the nerve membrane. Available evidence indicates that their principal action is to induce repetitive activity, which is due to a prolongation of the transient increase in sodium permeability of the nerve membrane during excitation.

\section{ACKNOWLEDGMENTS}

We thank Mr. R. Snijders for valuable cooperation. We are also indebted to Drs. M. Elliott and A. J. Gray and to Shell Research Ltd., Sumitomo Chemical Company, and W.H.O. for donation of pyrethroids. I'his work was supported by the Foundation for Medical Research FUNGO/ZWO and by Shell Internationale Research Mij.

\section{REFERENCES}

1. T. Narahashi, Effects of insecticides on excitable tissues, in "Insecticide Biochemistry and Physiology" (C. F. Wilkinson, Ed.), pp. 327-352. Plenum, New York, 1976.

2. W. Wouters and J. van den Bercken, Review: Action of pyrethroids, Gen. Pharmacol. 9, 387 (1978).

3. J. D. Doherty, Insecticides affecting ion transport, Pharmacol. Ther. 7, 123 (1979).

4. T. Narahashi, Effect of the insecticide allethrin on membrane potentials of cockroach giant axons, J. Cell. Comp. Physiol. 59, 61 (1962).

5. J. van den Bercken, L. M. $\Lambda$. $\Lambda$ kkermans, and J. M. van der Zalm, DDT-like action of allethrin in the sensory nervous system of Xenopus laevis, Fur. J. Pharmacol. 21, 95 (1973)

6. A. N. Clements and T. E. May, The actions of pyrethroids upon the peripheral nervous system and associated organs in the locust, Pestic. Sci. 8, 661 (1977).

7. K. Nishimura and T. Narahashi, Structureactivity relationships of pyrethroids based on direct action on nerve, Pestic. Biochem. Physiol. 8, 53 (1978).

8. T. A. Miller and M. E. Adams, Central vs. peripheral action of pyrethroids on the housefly nervous system, in "Synthetic Pyrethroids," ACS Symposium Series No. 42 (M. Elliott, Ed.), pp. 98-115, American Chemical Society, Washington, D.C., 1977.

9. M. Carlton, Some effects of cismethrin on the rabbit nervous system, Pestic. Sci. 8, 700 (1977).

10. J. van den Bercken, The action of allethrin on the peripheral nervous system of the frog, Pestic. Sci. 8, 692 (1977).

11. L. D. Leake, The action of (S)-3-allyl-2-methyl4 - ox oc yclopen t - - e n y l- ( IR) - trans chrysanthemate, (S)-bioallethrin, on single neurons in the central nervous system of the leech Hirudo medicinalis, Pestic. Sri. 8, 713 (1977).

12. M. E. Adams and T. A. Miller, Neural and behavioral correlates of pyrethroid and DDT-type poisoning in the house fly, Musca domestica. L., Pestic. Biochem. Physiol. 13, 137 (1980).

13. J. van den Bercken. A. B. A. Kroese, and L. M. A. Akkermans, Effects of insecticides on the sensory nervous system, in "Neurotoxicology of Insecticides and Pheromones" (T. Narahashi, Ed.), pp. 183-210, Plenum, New York, 1979.

14. J. van den Bercken and H. P. M. Vijverberg, Effects of insecticides on the sensory system of Xenopus, in "Insect Neurobiology and Pesticide Action (Neurotox. 79)," pp. 391-397. Society of Chemical Industry, London, 1980.

15. M. H. Evans, End-plate potentials in frog muscle exposed to a synthetic pyrethroid, Pestic. Biochem. Physiol. 6, 547 (1976).

16. W. Wouters, J. van den Bercken and A. van Ginneken, Presynaptic action of the pyrethroid insecticide allethrin in the frog motor end plate. Eur. J. Pharmacol. 43, 163 (1977).

17. T. Narahashi, Nerve membrane as a target of pyrethroids, Pestic. Sci. 7, 267 (1976).

18. J. van den Bercken and H. P. M. Vijverberg, Voltage clamp studies on the effects of allethrin and DDT on the sodium channels in frog myelinated nerve membrane, in "'Insect Neurobiology and Pesticide Action (Neurotox. 79)," pp. $79-85$, Society of Chemical Industry, London, 1980.

19. T. Narahashi and A. E. Lund, Giant axons as models for the study of the mechanism of action of insecticides, in "Insect Neurobiology and Pesticide Action (Neurotox. 79)," pp. 497-505, Society of Chemical Industry, London, 1980.

20. H. P. M. Vijverberg. J. M. van der Zalm. and J. van den Bercken, Similar mode of action of pyrethroids and DDT on sodium channel gating in myelinated nerves, Nature (London) 295, 601 (1982).

21. T. Narahashi and N. C. Anderson, Mechanism of excitation block by the insecticide allethrin applied externally and internally to squid giant axons, Toxicol. Appl. Pharmacol. 10, 529 (1967).

22. P. E. Burt and R. E. Goodchild, The action of pyrethroids in the insect nervous system I. Features of molecular structure associated with toxicity to cockroaches and to their giant fibre axons, Pestic. Sci. 8, 681 (1977).

23. R. D. Verschoyle and W. N. Aldridge, Structureactivity relationships of some pyrethroids in rats, Arch. Toxicol. 45, 325 (1980).

24. D. W. Gammon, M. A. Brown, and J. E. Casida, Two classes of pyrethroid action in the cockroach, Pestic. Biochem. Physiol. 15, 181 (1981). 
25. H. P. M. Vijverberg and J. van den Bercken, Frequency-dependent effects of the pyrethroid insecticide decamethrin in frog myelinated nerve fibres, Eur. J. Pharmacol. 58, 501 (1979).

26. T. Narahashi, Site and types of action of pyrethroids on nerve membrane, in "Pyrethroid Insecticides: Chemistry and Action" (J.E. Casida and M. Elliott, Eds.), pp. 15-17, Table Ronde Roussel-Uclaf, No. 37, Paris, 1980.

27. A. B. A. Kroese, J. M. van der Zalm, and J. van den Bercken, Frequency response of the lateral-line organ of Xenopus laevis, Pflügers Arch. 375, 167 (1978).

28. P. Görner, Untersuchungen zur Morphologie und Electrophysiologie des Seitenlinienorgans vom Krallenfrosch (Xenopus laevis Daudin), Z. Vergl. Physiol. 47, 316 (1963).

29. W. Salvisberg, G. Becker, and G. Voss, Insecticides and electrophysiological symptoms: A comparative study on a tarsal motor nerve of Locusta migratoria, Pestic. Biochem. Physiol. 13, 63 (1980).

30. H. P. M. Vijverberg and G. S. F. Ruigt, Influence of temperature on the interaction of pyrethroids with the sodium channels in the nerve membrane, Neurosci. Lett. Suppl. 7, S195, (1981).

31. H. P. M. Vijverberg, J. M. van der Zalm, R. G. D. M. van Kleef, and J. van den Bercken, Temperature and structure-dependent inter- action of pyrethroids with the sodium channels in frog node of Ranvier, Biochim. Biophys. Acta, in press.

32. A. Flock and D. M. K. Lam, Neurotransmitter synthesis in inner ear and lateral line sense organs, Nature (London) 249, 142 (1974).

33. R. P. Bobbin, S. C. Bledsoe Jr., D. M. Chical and D. N. Morgan, Comparative actions of glutamate and related substances on the Xenopus laevis lateral line. Comp. Biochem. Physiol. (C) 69,145 (1981).

34. P. R. Smith, The effect of cismethrin on the rat dorsal root potentials, Eur. J. Pharmacol. 66, 125 (1980).

35. L. M. A. Akkermans, J. van den Bercken, and M. Versluys-Helder, Comparative effects of DDT, allethrin, dieldrin and aldrin-transdiol on sense organs of Xenopus laevis, Pestic. Biochem. Physiol. 5, 451 (1975).

36. M. P. Osborne, The insect synapse: Structural functional aspects in relation to insecticidal action, in "Insect Neurobiology and Pesticide Action (Neurotox. 79)," pp. 29-40, Society of Chemical Industry, London, 1980.

37. A. J. Gray, T. A. Connors, H. Hoellinger, and Nguyen-Hoang-Nam, The relationship between the pharmacokinetics of intravenous cismethrin and bioresmethrin and their mammalian toxicity, Pestic. Biochem. Physiol. 13, 281 (1980). 